\title{
Validation of eAircraft battery simulation approach using field measurement data
}

\author{
T. Debreceni ${ }^{1}$, P. Szabó ${ }^{2}$, G. Gy. Balázs ${ }^{2}$ and I. Varjasi ${ }^{1}$ \\ ${ }^{1}$ Department of Automation and Applied Informatics, Budapest University of \\ Technology and Economics, Budapest, Hungary, \\ E-mails: tibor.debreceni@aut.bme.hu, istvan.varjasi@aut.bme.hu \\ ${ }^{2}$ Siemens Zrt., Budapest, Hungary, E-mails: szabo.peter@siemens.com, gergely.balazs@siemens.com
}

\begin{abstract}
Due to the specifically high power pulse and long duration energy requirements against lithium battery systems in electric aircrafts, the electrical design cannot be realized without sufficient modeling and simulation of such batteries considering the nonlinear behavior of cells and mission profiles. This paper presents an overview of the simulation approach and confirms the performance for using it as a design tool. The validation process of the models and simulation environment in MATLAB/Simulink includes error analysis investigating real measurement data recorded during flights of a fully electric aircraft.
\end{abstract}

\section{Key words}

Battery simulation, battery model, validation, identification, electric vehicle, electric aircraft, mission profile.

\section{Background}

Due to the nonlinear behavior, energy- and power densities of lithium battery cells used in electric vehicles compared to fuel, the energy efficiency is in the center of the focus next to safety especially in electric aircrafts. This can be handled and improved by energetic design of batteries considering the flight mission profiles from the very beginning phase of the design using simulation.

Published work [1] has presented a simulation environment in MATLAB/Simulink suitable for iterative sizing of lithium-based battery considering optional mission profile inputs as system requirements. For the better understanding of the actual validation work, the background is summarized here.

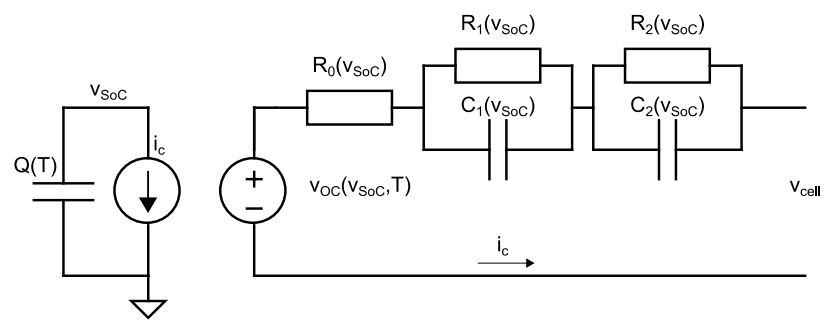

Fig. 1. 2-RC branch electrical lithium cell model.
The response of the cell voltage $\left(\mathrm{v}_{\text {cell }}\right)$ to constant or pulsed cell current $\left(i_{c}\right)$ can be modeled using an equivalent circuit model shown in a second-order form in Fig. 1.

$$
\operatorname{SoC}(t)=\operatorname{SoC}(0)+\frac{1}{Q(T)} \int_{0}^{t} i_{c}(t) d t
$$

where $i_{c}$ is positive when the cell is charged and negative when discharged.

The series resistor, $\mathrm{R}_{0}$, accounts for the ohmic losses in the cells and the two RC pairs, $\mathrm{R}_{1} \mathrm{C}_{1}$ and $\mathrm{R}_{2} \mathrm{C}_{2}$ account for the diffusive, in other words, transient losses [3], [6], [7], [8].

The number of RC pairs can be chosen to fit to the experimental data, but at the cost of greatly increased time for the identification process of the model parameters. The 2-RC branch model is widely accepted as the practically best trade-off between model accuracy and complexity [2], [3], [8]. So the envelopes of the model discharge curves fit to the measurement data while the cost of the identification process is kept acceptably low.

The paper is so organized. At first, a model refinement is discussed based on the work after publishing the first results in [1]. After that, we describe the model validation process including the used Simulink environment and the measurement data categories in section 3. After a short review on the investigated model error types, in section 4, the evaluation method is discussed in order to prescribe what we can accept or what should be adapted into the model. In section 5 the results are introduced in characteristics and numerical forms, as well. Section 6 deals with the conclusions and further steps.

\section{Model refinement}

Theoretically, all parameters vary with current, temperature, SoH, SoC, etc [4], [8]. Battery model using such topology can be effectively identified to provide parameters as multivariable nonlinear functions [2], [4], [8]. However, in the described model the priority of some 
parameter considerations are boosted, others are decreased or totally neglected in respect of the application and regarding the model operation on battery pack level defined as in [1].

A measurement-based identification process has been done in laboratory in order to achieve sufficient I-V performance for the determination of voltage response to pulse and constant loads, for runtime and efficiency estimations during mission profiles [1].

Without going into details of the identification, a dependency graph of the realized model is shown in Fig. 2, where a parameter at the end of the arrow is dependent from a parameter at the base of the arrow.

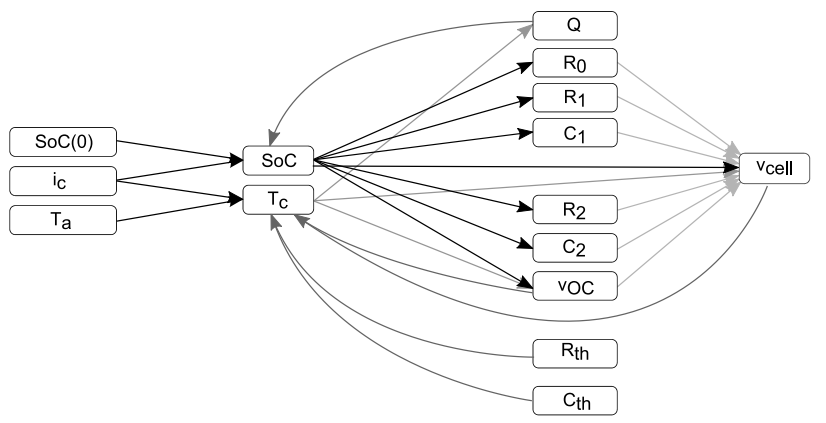

Fig. 2. Dependency graph of the model parameters.

The cell temperature $\left(T_{c}\right)$ is determined using a one-time constant equivalent thermal model of the battery cell [5] shown in Fig 3.

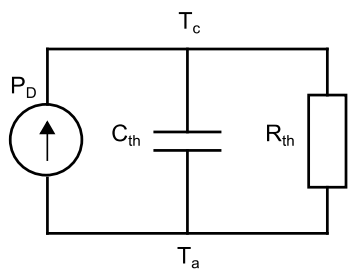

Fig. 3. Used thermal model of the cell.

$\mathrm{C}_{\text {th }}$ capacitor represents the heat capacitance of the cell, and $\mathrm{R}_{\mathrm{th}}$ resistor represents the resulting thermal junction between the cell internal heat generation and the ambient medium $\left(\mathrm{T}_{\mathrm{a}}\right)$ through the cell surface. Therefore, the thermal behavior can be described as in (2).

$$
\begin{gathered}
P_{D}=\left|\left(v_{o c}-v_{c e l l}\right) i_{c}\right| \\
T_{c}=T_{a}+\frac{1}{C_{t h}} \int_{0}^{t}\left(P_{D}-\frac{T_{c}-T_{a}}{R_{t h}}\right) d t
\end{gathered}
$$

Note that since heat radiation effect of the cells and generally inside the battery system, and cooling medium, like airflow are not assumed, one cell temperature can be considered as a resulting temperature of the battery pack.

\section{Validation process and environment}

Regarding the model operation, the load current of the battery pack measured in the drive system is required as an input of the battery pack model in the validation environment. Concerning the dependency graph in Fig. 2, ensuring the ambient temperature and initial SoC for the model are effortless. Latter is done by applying the inverse function of the open circuit voltage in SoC domain for the unloaded battery pack. We assume that there is no deviation between the parallel connected cell blocks inside the battery pack, therefore one resulting initial SoC can be determined.

However, concerning the measured pack current $\left(i_{p}\right)$ as an input, it turns out, that all parameters in the model strongly depends on it and its measurement error. For example, the SoC estimation based on (1) directly accumulates the measurement error in the current data. Therefore, a moving average filter is applied on the sampled current measurement data.

The validation setup in MATLAB/Simulink can be seen in Fig. 4, where the mission profiles are imported into a signal builder.

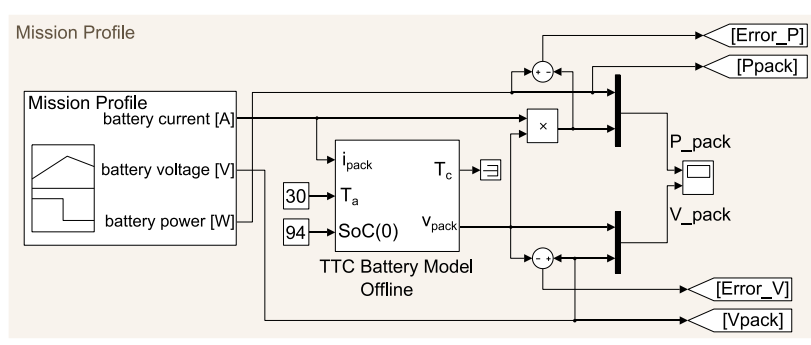

Fig. 4. Battery model validation setup.

The signal builder already consists of the filtered battery current. Since the battery voltage is used for error analysis, here a moving average filter is applied on the sampled battery voltage measurement data. The ambient temperature of the whole pack is assumed to be the maximum value of measured cell temperatures once a day before the first flight.

Essentially, mission profile is a power curve in time domain, and used in the energetic design of the battery as a requirement specification. The validation work is, however, focusing mainly on the voltage errors instead of power errors. Voltage is a direct model output - instead of an inherited power - and exist as direct measurement data from the experiments.

62 mission profiles are used for validation in this study containing two different types. First will be referred to as "routine mission profile" as can be seen in Fig. 5.

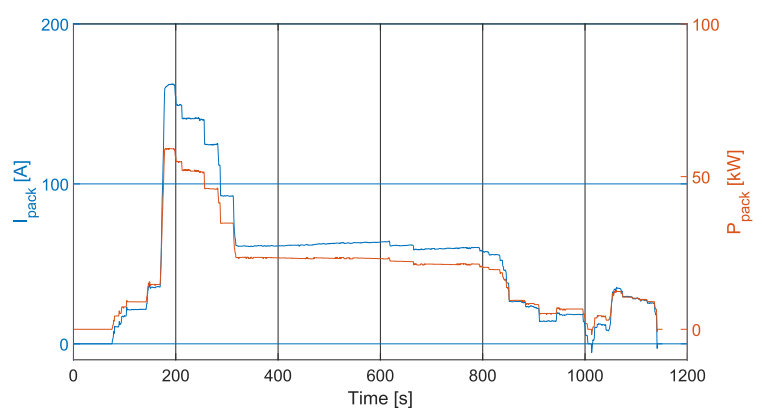

Fig. 5. Routine mission profile example.

In this case, there is only one high power pulse at takeoff, from that, decreasing power is needed until the cruising phase, when the power is roughly constant. 
The other type is called "touch-and-go", which represents short training cycles shown in Fig. 6.

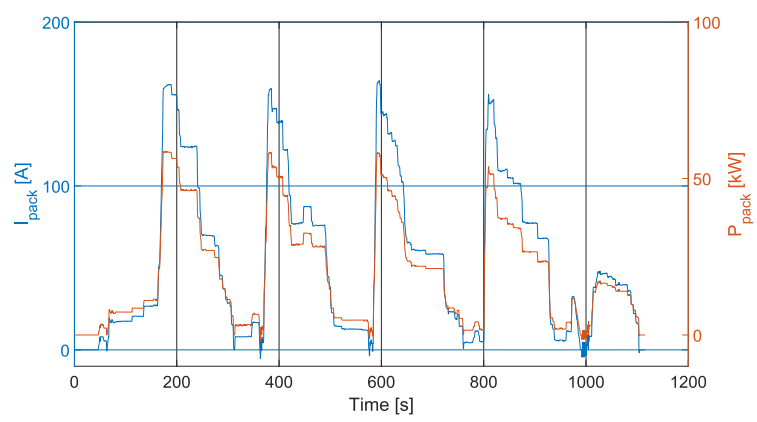

Fig. 6. Touch and go mission profile example.

\section{Error analysis method}

In order to evaluate the accuracy of model predictions, the goodness of fit between the measurement and modeled data is needed to determine. These model accuracy metrics are chosen considering the nonlinearity of the data due to the nonlinear battery cell behavior.

The main focus of the error analysis is the voltage performance. At first, the voltage data of the model and the measurement are plotted in the same graph in order to make some characteristic review whether the domains and data lengths are sufficient. Then the relative voltage errors are obtained and plotted in time domain based on (3).

$$
e_{v, i}=\frac{v_{d, i}-v_{m, i}}{v_{d, i}}[\%]
$$

where $v_{d, i}[V]$ is the $i^{\text {th }}$ measured voltage data and $v_{m, i}[V]$ is the $\mathrm{i}^{\text {th }}$ voltage output of the model.

Note that there could be offset and rate error in the voltage measurement, which could not be filtered out by moving average filter and cannot be identified and eliminated by the error analysis.

After the first review, different type of histograms are used for error representation. Every type is handled separately for routine and touch-and-go profiles.

\section{A. Type I}

It consists of $e_{v, i}$ values per mission profiles, so one histogram will show the relative error frequency considering one certain mission profile.

\section{B. Type II}

Maximum values of $\left|e_{\mathrm{v}, \mathrm{i}}\right|$ (absolute) are taken from each mission profile and plotted in one histogram. This method will show us the frequency of the worst case errors for all samples of profiles. We can prescribe a maximum frequency number that is prohibited to be overstep in the total domain of relative error.

\section{Type III}

Type I histogram information are summarized in one histogram. We can prescribe a range of relative errors, e.g.
$2 \sigma$, which will determine $N_{v}$ samples belonging to this range in the histogram, and then $\mathrm{N}_{\mathrm{v}} / \mathrm{N}$ can be considered in the evaluation process with a minimum value of, for example, $90 \%$.

In order to analyze quantitatively the accuracy of model outputs, more error types were considered for this purpose, especially R-square, root-mean-square error (RMSE), normalized root-mean-square error (NRMSE), Pearson correlation coefficient (PCC) and Nash-Sutcliffe efficiency coefficient (NSE) methods.

The aim was to provide a metric that is capable of describing effectively the goodness of fit for the nonlinear model and data. We found that using two of those methods will allow us to evaluate the model and simulation approach more effectively. Therefore, we have chosen RMSE and NSE. Determination of RMSE is done by (5), in turn, NSE can be described as in (6).

$$
R M S E=\sqrt{\frac{\sum_{i=1}^{N}\left(v_{d, i}-v_{m, i}\right)^{2}}{N}}[V]
$$

NRMSE is a non-dimensional form of the RMSE, which is often used when comparing RMSE with different units. Here we do not need to use NRMSE, each dataset is in voltages.

$$
N S E=1-\frac{\sum_{i=1}^{N}\left(v_{d, i}-v_{m, i}\right)^{2}}{\sum_{i=1}^{N}\left(v_{d, i}-\bar{v}_{d}\right)^{2}}
$$

where $\bar{v}_{d}[\mathrm{~V}]$ is the average value of measured voltage data. NSE can range from $-\infty$ to 1 , where 1 corresponds to a perfect match between model and measurement data, 0 indicates that the model predictions are as accurate as the mean of the measurement data. An efficiency less than zero $(-\infty<\mathrm{NSE}<0)$ occurs when even the measured mean is better predictor than the model. Essentially, the closer the NSE is to 1, the more accurate the model is [9]. Note that MATLAB uses the Modified NSE (MNSE) as an integrated function for model validation against experimental data. MNSE uses absolute errors instead of quadratic errors.

RMSE and NSE will be determined in case of all mission profiles and summarized in histograms separately. At the end, we can have important conclusions considering fitted curves for the summarized RMSE and NSE histograms, as well.

\section{Results and evaluation}

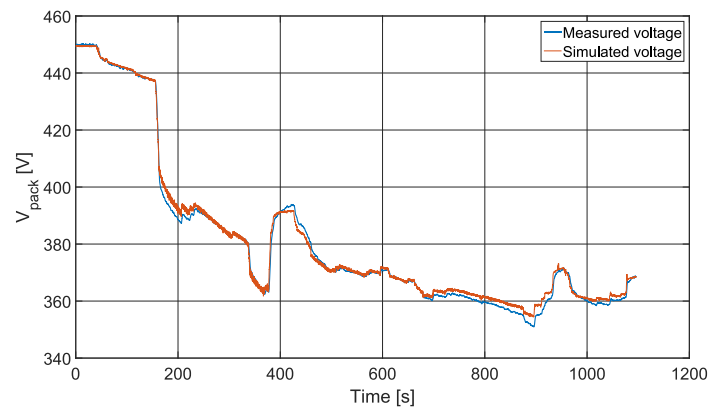

Fig. 7. Review on example voltage curve in time. 
As it was mentioned before, the first step was to make short but important check of the validation environment operation and data conditioning by review. The forms and characteristics of the voltage curves in time can be exampled as shown in Fig. 7. The calculated relative voltage error for this example is shown in Fig. 8.

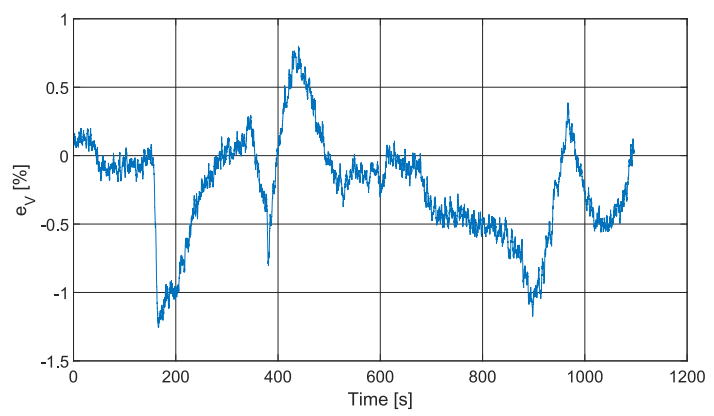

Fig. 8. Review on example rel. voltage error in time.

Based on the preliminary results and expectations, the curves show proper kind of operation.

Then, the relative voltage errors, $\mathrm{e}_{\mathrm{v}, \mathrm{i}}$, are summarized and evaluated. The above described Type I, II and III histograms are shown from Fig. 9 to Fig. 13. In case of each histogram also the fitted standard distribution curves are plotted and the mean values are displayed. These show us a very good model performance, the mean values for Type I and Type III are significant low to conclude that this model is capable for battery simulation.

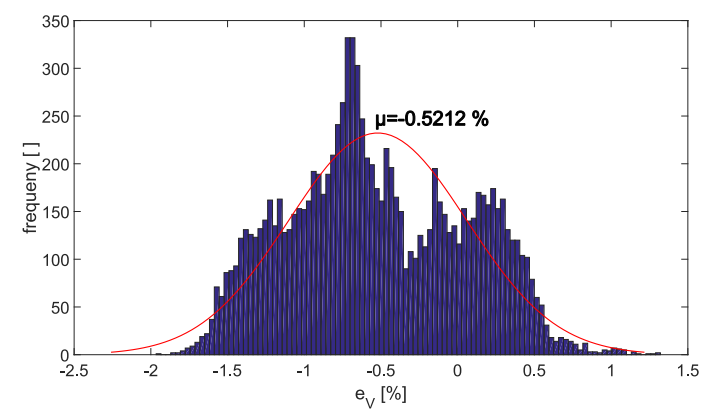

Fig. 9. Type I histogram for one routine mission profile.

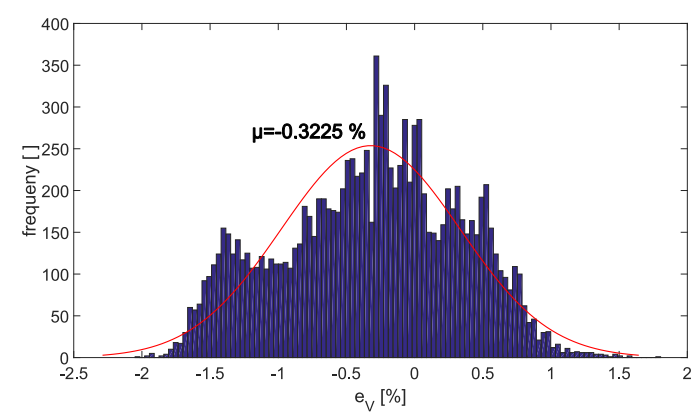

Fig. 10. Type I histogram for one touch-and-go mission profile.

Even in case of summarizing the worst results in Type II histograms, the mean value is about $4 \%$, which is absolutely acceptable in simulations, so the expected worst case error is still low enough to predict I-V performance on system level.

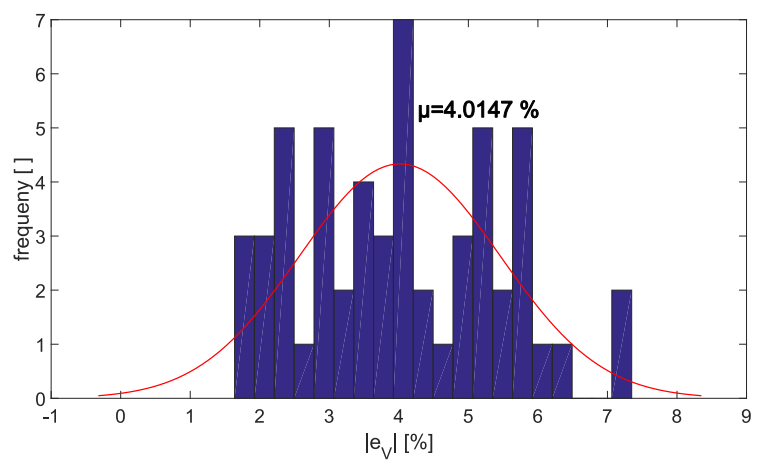

Fig. 11. Type II histogram for routine mission profiles.

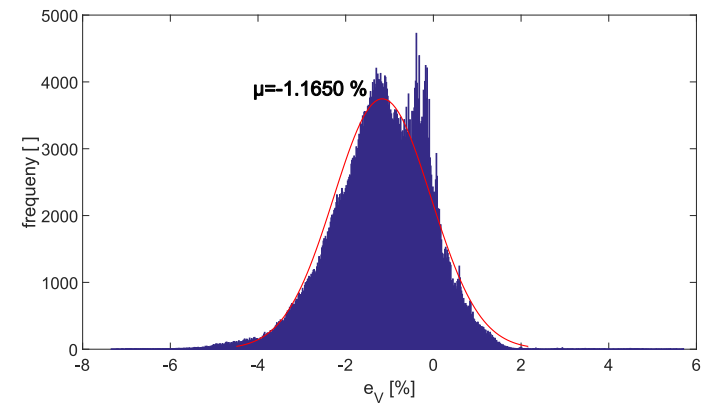

Fig. 12. Type III histogram for routine mission profiles.

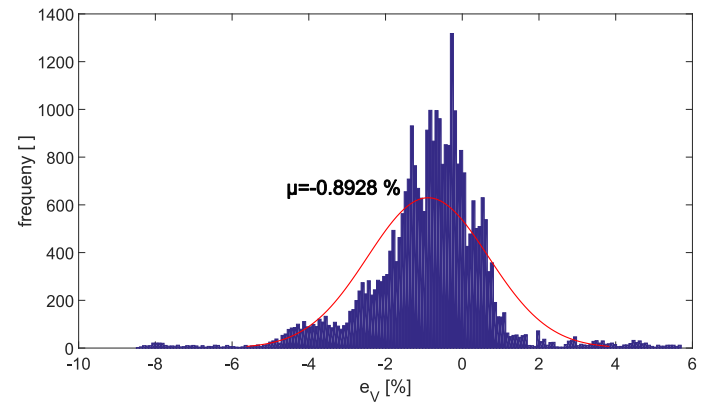

Fig. 13. Type III histogram for touch-and-go mission profiles.

Considering a focus range of $2 \sigma$ in Type III, $\mathrm{N}_{\mathrm{v}} / \mathrm{N}$ ratio of $95.7 \%$ for routine and $93.9 \%$ for touch-and-go mission profiles are obtained. Hereby, we can state that the prescribed $90 \%$ is definitely fulfilled in both cases.

After evaluating the results represented by the above histograms, the numerical measures of goodness of fit are determined based on (5) and (6). At first, here the worst and the best results of NSE and RMSE are selected from the validation test runs using routine mission profiles. This gave us only an insight by review on the extremes that can be seen in Table I.

Table I. - Extremes of RMSE and NSE for routine mission profiles.

\begin{tabular}{|l|c|c|}
\hline & Worst result & Best result \\
\hline RMSE [V] & 9.3454 & 1.4339 \\
\hline NSE [] & 0.8255 & 0.996 \\
\hline
\end{tabular}

One can conclude that the error variance cannot be too large regarding the extremes. Therefore, the total RMSE and NSE are calculated for all routine mission profiles and plotted in Fig. 14 and Fig. 15. 


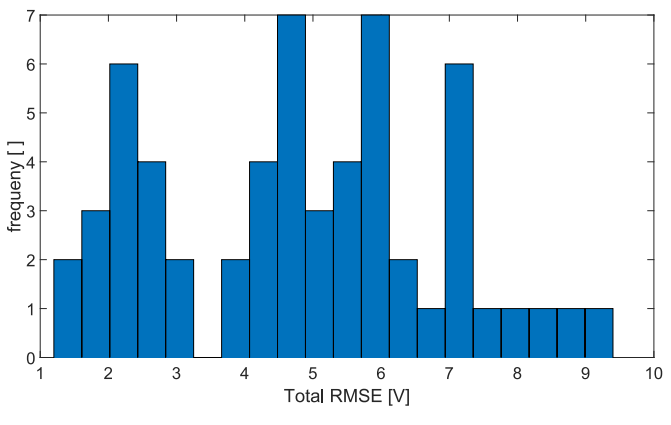

Fig. 14. Total RMSE for routine mission profiles.

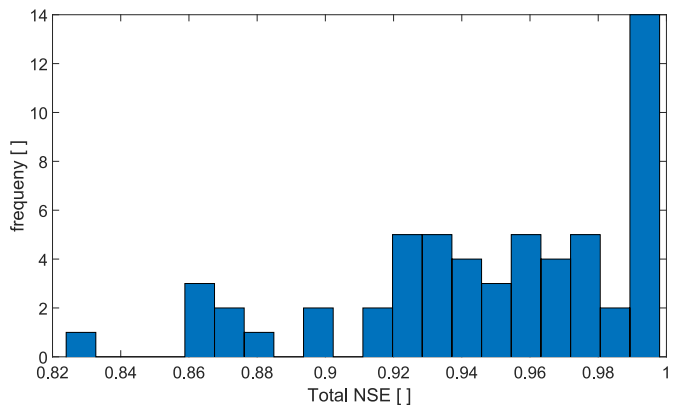

Fig. 15. Total NSE for routine mission profiles.

Regarding the total RMSE, the most frequent errors are within a certain error tolerance between $2 \mathrm{~V}$ and $8 \mathrm{~V}$. Considering the total operating voltage of the battery system, which is [270V;450 V], these RMSE values are definitely acceptable.

In Fig. 15 one can clearly see that, however, there are samples also around 0.87 and from 0.92 to 0.98 , the most frequent total NSE values can be found above 0.99 . This is a final numerical provement of the goodness of fit, which allows us to state that the model performance is good enough for design. The design predictions will be fit in the range of [0.8255;0.996], but most probably above 0.99 .

\section{Conclusion}

In this paper, a battery model and simulation approach using for iterative energetic design is validated. The short overview of the previously published model with the discussion of the model refinement are presented. The validation environment and setup is examined. Different types of errors and categories for analysis and representation are chosen and introduced. The model performance is analyzed by showing and evaluating the results using more methods.

As it is confirmed by the presented results, the conclusion is that the battery model and simulation approach can be used for effective energetic design of battery systems considering also rapid iterations with mechanical requirements, like weight and volume. The described routine and touch-and-go mission profiles were different enough to allow us the validation with high sample numbers but using more characteristic variants, as well.

The error tolerances show us that this simulation environment allow us not only to observe, but to analyze even the battery runtime and transient behavior in details for a sufficient and effective energetic design of such safety- and mission-critical systems.

\section{Acknowledgement}

The authors are very grateful to Dr. Frank Anton for providing research and development directions of battery systems. Many thanks to Richard Szabó for submitting the measurement data of eFusion flights. Authors are also much obliged to Dr. Holger Wolfschmidt for his all-time support in battery topics.

\section{References}

[1] T. Debreceni, G. Gy. Balázs and I. Varjasi, "Mission Profile-Oriented Design of Battery Systems for Electric Vehicles in MATLAB/Simulink®", International Conference on Renewable Energies and Power Quality (ICREPQ'16), Madrid, Spain, May 2016, Renewable Energy and Power Quality Journal (RE\&PQJ) Conference Proceedings.

[2] Chen, M., Rincón-Mora, G.A: "Accurate Electrical Battery Model Capable of Predicting Runtime and I-V performance", IEEE Transactions on energy conversion, Vol. 21 No. 2, 2006, p.504-511.

[3] Shifei Yuan, Hongjie Wu and Chengliang Yin, "State of Charge Estimation Using the Extended Kalman Filter for Battery Management Systems Based on the ARX Battery Model”, Energies 2013, 6, 444-470.

[4] Long Lam: "A Practical Circuit-based Model for State of Health Estimation of Li-ion Battery Cells in Electric Vehicles", Master of Science Thesis, University of Technology Delft, 2011.

[5] Tibor Debreceni, Péter Szabó, Gergely György Balázs, István Varjasi, FPGA-synthesizable Electrical Battery Cell Model for High Performance Real-time Algorithms. Periodica Polytechnica Electrical Engineering and Computer Science, Vol. 60, No. 3, pp. 171-177, 2016. DOI:10.3311/PPee.9398

[6] He, H.; Xiong, R.; Fan, J., "Evaluation of lithium-ion battery equivalent circuit models for state of charge estimation by an experimental approach", Energies 2011, 4, 582-598.

[7] Rui Xiong, Xianzhi Gong, Chunting Chris Mi, Fengchun Sun: "A robust state-of-charge estimator for multiple types of lithium-ion batteries using adaptive extended Kalman filter", Journal of Power Sources, vol. 243, 2013, 805-816.

[8] H. Rahimi-Eichi and M.-Y. Chow, "Adaptive parameter identification and State-of-Charge estimation of lithiumion batteries," presented at the 38th Annual Conference on IEEE Industrial Electronics Society (IECON 2012), IEEE, pp. 4012 - 4017, Montreal, QC, Canada, 2012

[9] Stefano Marsili-Libelli, "Environmental Systems Analysis with MATLAB ${ }^{\circ}$ ", side 112., CRC Press, 2016, ISBN 9781498706353 